\title{
Correlation between nasal potential difference measurements, genotype and clinical condition in patients with cystic fibrosis
}

\author{
L.P. Ho*, J.M. Samways*, D.J. Porteous**, J.R. Dorin**, A. Carothers **, \\ A.P. Greening*, J.A. Innes*
}

Correlation between nasal potential difference measurements, genotype and clinical condition in patients with cystic fibrosis. L.P. Ho, J.M. Samways, D.J. Porteous, J.R. Dorin, A. Carothers, A.P. Greening, J.A. Innes. (CERS Journals Ltd 1997.

ABSTRACT: In cystic fibrosis (CF), the clinical condition of patients correlates poorly with genotype. One possible explanation is that clinical status is influenced by net preserved chloride secretion rather than the CF mutation. We tested the relationships between residual chloride secretion, as measured by nasal potential difference (PD) and the type of mutation (genotypes expressing apical cystic fibrosis transmembrane conductance regulator (CFTR) protein versus those that do not) and clinical status.

Twenty two CF patients (mean age 25.7 yrs, 11 females and 11 males, mean forced expiratory volume in one second (FEV1) $53.1 \%$ of predicted) with defined genotypes were recruited. Nasal PD was measured using a standard protocol involving the perfusion of the nasal epithelium with a sodium channel blocker (amiloride), followed by a solution of low chloride and finally with isoprenaline.

Patients with apical CFTR protein showed higher residual chloride secretion than those without (amiloride to isoprenaline value of 4.59 and $0.56 \mathrm{mV}$, respectively, $p=0.01$ ). There was no correlation between mutation type and clinical condition. When these patients were recategorized as "high" $(>10 \mathrm{mV}$ amiloride to isoprenaline response) or "low" (10 $\mathrm{mV}$ or less) chloride secretors, we found that the former group had a significantly higher FEV1 (67.7 versus $48.3 \%$ pred) and a better pulmonary radiological score (4.14 versus $\mathbf{7 . 0 7}$, by Northern scoring system).

These results suggest that some cystic fibrosis patients, regardless of genotype, have an ability to secrete chloride when stimulated with chloride secretatagogues, and this is correlated with a better lung function. These results also have implications for the use of potential difference measurements in novel cystic fibrosis transmembrane conductance regulator replacement trials. Eur Respir J 1997; 10: 2018-2022.
*Scottish Adult Cystic Fibrosis Unit, Western General Hospital, Crewe Road, Edinburgh. ** MRC Human Genetics Unit, Western General Hospital, Edinburgh.

Correspondence: L.P. Ho

Scottish Adult Cystic Fibrosis Unit

Western General Hospital

Crewe Road

Edinburgh EH4 2XU

UK

Keywords: Chloride

cystic fibrosis

genotype

lung function

nasal potential difference

Received: December 301996

Accepted after revision June 291997
The major ion transport abnormality in cystic fibrosis (CF) airway epithelium results from the lack of a correctly localized and fully functioning cystic fibrosis transmembrane conductance regulator (CFTR) protein. This protein functions primarily as a chloride channel but also regulates sodium and other non-CFTR-mediated chloride conductive pathways [1]. Hence, in CF patients, there is an inability to secrete chloride ions from the apical surface of the cell and an enhanced sodium absorption, contributing to dehydrated, viscous secretions. In the lungs, the consequence is chronic bacterial colonization and repeated infections leading to bronchiectasis and fibrosis. Respiratory failure is eventually the cause of death for most of these patients.

Over 500 mutations of the CFTR gene have been characterized to date. Most of these mutations can be divided into either mislocalization of a dysfunctional or partially functioning CFTR product or a properly localized but dysfunctional CFTR protein. Numerous groups have attempted to correlate the severity of the disease with genotype, but often the association is poor. Within a genotype, the pulmonary function can be widely variable. Homozygotes for $\Delta F 508$ mutation have been found to have a greater incidence of pancreatic insufficiency, worse lung function and higher mortality compared to other genotypes $[2,3]$ while isolated mutations such as $A 455 E$ are associated with a mild clinical course [4]. However, a cohort study in Italy showed that pulmonary status in $8.5-10 \mathrm{yr}$ old $\Delta F 508$ homozyotes was no different from that in other mutations [5]. Indeed, the conclusion from a large phenotype genotype consortium [6] was that the only prognostic value of genotypic information is for the prediction of pancreatic sufficiency.

All this suggests that within any one genotype there is variation in clinical condition between patients and the phenotype of a patient may be influenced by other transcriptional and/or post-translational effects which determine the net function of the mutant protein, or by variations between individuals in the activity of other parallel ion transport systems. VeEze et al. [7] showed that in rectal epithelium of CF patients, residual chloride secretion, determined by Ussing chamber short circuit measurements, correlated with pancreatic sufficiency and age of presentation of disease. A less invasive 
method of measuring chloride secretion is to measure the transepithelial potential difference (PD) in the nose. In this method, the nasal epithelium is first perfused with amiloride which blocks sodium absorption, resulting in reduction of the negativity of the PD. This provides an electrical gradient for the secretion of chloride. Chloride secretion is further stimulated by the biochemical gradient provided by a low chloride solution and then by isoprenaline which stimulates the cyclic adenosine monophosphate (cAMP)-dependent pathway of secretion. In CF, the inability to secrete chloride can be demonstrated by a lack of change in PD in response to isoprenaline and low chloride solution [8-10].

We examined the relationship between nasal PD measurement, clinical condition and genotype in $\mathrm{CF}$ patients in order to evaluate nasal PD as a marker of CFTR function and an index of clinical condition in patients with $\mathrm{CF}$.

\section{Methods}

\section{Subjects}

The nasal PD protocol was approved by the local Ethics Committee and patients gave informed consent.

Twenty two cystic fibrosis patients $(11$ males, 11 females, mean (range) age 25.7 (17-45) yrs) of fully defined genotype were recruited. All patients attend the Scottish Adult CF Clinic in Edinburgh or the West of Scotland CF Clinic in Glasgow. Patients on nasal medication, those with nasal polyps, allergic rhinitis or nasal surgery in the year preceeding the measurement were excluded. Measurements during upper or lower respiratory tract infections were also excluded. Lung function (mean of two most recent measurements of percentage predicted forced expiratory volume in one second (FEV1) when stable) in these patients ranged $20-97 \%$, but all patients were clinically stable during the study.

All patients had at least four measurements separated by at least 5 days. In certain patients of interest (high secretors, see below) measurements were made on up to 12 occasions. For comparison, 12 non-CF patients were also tested on between one and six occasions. There were six males and six females (mean (range) age, 34.5 (26-41) yrs). The same exclusion criteria were applied as for $\mathrm{CF}$ patients and measurements were made under the same conditions.

\section{Nasal PD measurement}

Nasal PD measurements were performed using a method as previously described by MiDDLETON et al. [9] and used routinely by us [11]. Briefly, a modified Foley size 8 paediatric urinary catheter filled with diluted electrode gel and containing a silver/silver chloride electrode was used. This electrode was connected via an isolated pre-amplifier and digitizer (Logan-Sinclair, Kent, UK) to a palm top computer (Psion) which recorded the voltage every second. A reference electrode was attached to a lightly abraded area of the forearm.

The catheter was passed gently along the floor of the nose and positioned at the point of maximal negative voltage nearest the posterior end of the nasal floor. The patient held the catheter and maintained this position over 20-30 min. Recordings were made every second while the nostril was perfused $\left(4 \mathrm{~mL} \cdot \mathrm{min}^{-1}\right)$ first with the diluent (buffered Kreb's) solution, followed by diluent with $100 \mu \mathrm{M}$ of amiloride (to block sodium current), then, in order to drive chloride secretion, amiloride (100 $\mu \mathrm{M}$ ) in low chloride diluent (low chloride solution) and finally isoprenaline $(10 \mu \mathrm{M})$ and amiloride $(100 \mu \mathrm{M})$ in low chloride diluent (isoprenaline solution) to stimulate CFTR-dependent chloride secretion. Each solution perfused the nostril for a minimum of $180 \mathrm{~s}$ or until a steadyvoltage plateau of at least $1 \mathrm{~min}$ had been achieved. The mean for the last $60 \mathrm{~s}$ (excluding the final $5 \mathrm{~s}$ before a change of solution) was taken as the final reading for each solution.

For ease of reading, "amiloride to low chloride", refers to differences in nasal PD values from the plateau achieved during amiloride in buffered Krebs Hepes solution to that achieved during amiloride in low chloride solution. Similarly, "amiloride to isoprenaline" refers to net change between amiloride in buffered Krebs Hepes solution and amiloride and isoprenaline in low chloride solution

This method provides a profile of how nasal PD values change with different perfusates. The point of maximal CFTR stimulation was taken as the final plateau reading achieved during perfusion with isoprenaline in low chloride and amiloride solution. Hence the amiloride to isoprenaline difference represents the maximal chloride secretion achieved using this protocol.

\section{Data analysis}

Patients were divided into two groups, according to genotype: 1) mutations that fail to generate significant apical membrane protein $(\triangle F 508 / \Delta F 508, \Delta F 508 / W 1282 X$, $\triangle F 508 / Q 493 X)$ and 2) mutations where gene product is present in the apical membrane $(\Delta F 508 / G 551 D, \Delta F 508 /$ $A 455 E, \Delta F 508 / R 117 H, G 551 D / G 551 D)$ [12]. There were 143 measurements in $22 \mathrm{CF}$ patients and 61 in normal subjects. Each patient contributed between 4-8 individual nostril measurements to the genotype/PD analysis. There were 15 patients in genotype Group 1, contributing 97 PD measurements, and seven patients in Group 2, contributing 46 PD measurements. Analysis of variance allowing for all contributing sources of variation (e.g. difference in number of measurements per patient, number of patients per group) was carried out on: 1) baseline; 2) change from amiloride to low chloride; and 3) change from amiloride to isoprenaline values.

In order to formulate a representative clinical picture for each patient, the following data were recorded from the case notes of the patients: age; mean of the two most recent values of FEV1 and forced vital capacity (FVC) when the patient was stable (both as \% pred); highest body mass index (BMI) within 6 months of PD measurement; pancreatic sufficiency; absence or presence of Pseudomonas colonization at time of PD measurement; number of intravenous antibiotic courses administered for infective exacerbations of pulmonary disease within 12 months of PD measurements; and the Northern radiological score [13] on chest radiographs within 1 month of the measurements. The latter was scored by two physicians according to standard protocol, where an increasing score indicates worsening radiological appearance, up to a maximum score of 16 . 


\section{Results}

\section{Comparison of $P D$ measurements in $C F$ and non-CF subjects}

The measurements in non-CF subjects and $\mathrm{CF}$ patients showed mean baseline values of 14.9 and 43.3 $\mathrm{mV}$, respectively, with mean amiloride to low chloride values of 18.6 and $3.2 \mathrm{mV}$ and amiloride to isoprenaline values of 29.0 and $2.1 \mathrm{mV}$ (table 1).

Table 1. - Comparison of mean baseline and stimulated potential difference (PD) values obtained in normal and cystic fibrosis (CF) patients

\begin{tabular}{|c|c|c|c|c|c|}
\hline & Subjects & $\begin{array}{c}\text { Mean } \\
\mathrm{mV}\end{array}$ & SD & SEM & $\begin{array}{c}95 \% \\
\text { confidence } \\
\text { interval }\end{array}$ \\
\hline \multicolumn{6}{|l|}{ CF patients } \\
\hline Baseline & 22 & 43.3 & 10.5 & 0.9 & $22.5-64.1$ \\
\hline Amiloride-Low Cl & 22 & 3.2 & 5.6 & 0.5 & $-7.9-14.3$ \\
\hline Amiloride-Isoprenaline & ne 22 & 2.1 & 7.1 & 0.6 & $-12.0-16.2$ \\
\hline Low Cl-Isoprenaline & 22 & -1.0 & 3.8 & 0.3 & $-8.5-6.5$ \\
\hline \multicolumn{6}{|l|}{ Non-CF patients } \\
\hline Baseline & 12 & 14.9 & 6.9 & 0.9 & $1.1-28.7$ \\
\hline Amiloride-Low $\mathrm{Cl}$ & 12 & 18.6 & 11.2 & 1.4 & $-3.8-41.0$ \\
\hline Amiloride-Isoprenaline & ne 12 & 29.0 & 12.6 & 1.6 & $3.8-54.2$ \\
\hline Low Cl-Isoprenaline & 12 & 10.3 & 5.4 & 0.7 & $-0.5-21.1$ \\
\hline
\end{tabular}

By convention, negative signs are ignored although all PD values are negative. Mean values are of all values from both nostrils in each patient.

Table 2. - Difference in mean values of baseline potential difference $(\triangle P D)$ and stimulated values between two genotype groups

\begin{tabular}{lccc}
\hline Genotype & $\begin{array}{c}\text { Baseline PD } \\
\mathrm{mV}\end{array}$ & $\begin{array}{c}\Delta \mathrm{PD} \text { from } \\
\text { amiloride to } \\
\text { low chloride } \mathrm{mV}\end{array}$ & $\begin{array}{c}\Delta \mathrm{PD} \text { from } \\
\text { amiloride to } \\
\text { isoprenaline } \mathrm{mV}\end{array}$ \\
\hline Group 1 & $43.30(10.70)$ & $2.05^{+}(5.00)$ & $0.56^{\dagger}(6.13)$ \\
Group 2 & $43.51(10.00)$ & $4.65^{+}(6.10)$ & $4.59^{\dagger}(7.9)$
\end{tabular}

Results are mean (SD), weighted statistically to allow for different number of patients between groups. Group 1: no or minimal apical cystic fibrosis transmembrane regulator (CFTR) protein, $\mathrm{n}=97$ from 15 patients. Group 2: apical CFTR protein present, $\mathrm{n}=46$ from seven patients. ${ }^{+}: \mathrm{p}=0.04 ;{ }^{\dagger}: \mathrm{p}=0.01$, analysis of variance (ANOVA), for comparison between groups.

Table 3. - Comparison of clinical characteristics between patients in Group 1 (no or minimal apical CFTR protein) and Group 2 (apical CFTR protein present).

\begin{tabular}{lll}
\hline & Group 1 & Group 2 \\
\hline Patients n & 15 & 7 \\
Amiloride to & $0.56(6.13)$ & $4.59(7.9)$ \\
$\quad$ isoprenaline value mV & $25.4(6.7)$ & $31.6(5.1)$ \\
Age yrs & $52.3(18.8)$ & $61.0(19)$ \\
FEV1 \% pred & $68.3(15.6)$ & $76.6(10.2)$ \\
FVC \% pred & 60 & 71 \\
$\begin{array}{l}\text { Patients with P. aeruginosa } \\
\quad \text { colonization \% }\end{array}$ & 3.4 & 2.4 \\
$\begin{array}{l}\text { i.v. antibiotics for } \\
\text { infective exacerbation }\end{array}$ & & \\
$\quad$ in last 12 months n & & $5.3(1.5)$ \\
Northern radiological score & $6.6(2.2)$ &
\end{tabular}

Values are presented as mean, and SD in parenthesis, unless otherwise stated. There were no significant differences between groups ( $\mathrm{p}>0.05$, t-test). CFTR: cystic fibrosis transmembrane conductive regulator; FEV1: forced expiratory volume in one second; FVC: forced vital capacity.

\section{Effect of genotype group on nasal PD}

Dividing the patients into genotype groups as mentioned above, we found that there were statistically significant differences in the amiloride to low chloride and the amiloride to isoprenaline measurements between the two groups (table 2)

For all three measurements, Group 2 (in whom apical CFTR protein was retained) showed a larger capacity to secrete chloride compared to Group 1.

\section{Effect of genotype group on clinical parameters}

Despite the statistically significant effect on CFTRrelated PD measurements, we found no statistically significant difference in clinical parameters between patients with mutations that lack (Group 1) and those that retained an apical CFTR protein (Group 2) (table 3).

\section{Correlation between chloride secretion and clinical para- meters}

In view of the above results, we examined whether chloride secretion is a better determinant of clinical status of CF patients than the genotype group. We divided the patients into two groups according to their ability to secrete chloride in response to isoprenaline perfusion (amiloride to isoprenaline values). An amiloride to isoprenaline value of $\leq 10 \mathrm{mV}$ was arbitrarily chosen as representative of "low" chloride secretion while patients with values $>10 \mathrm{mV}$ were classed as "high secretors". We found that patients in the "high" secretor group had a significantly better FEV1 \% pred (table 4 and fig. 1).

The two subjects with the highest chloride-secreting nasal PD responses were pancreatic sufficient while the rest were pancreatic insufficient.

Table 4. - Comparison of clinical characteristics between patients with "low" or no stimulated chloride secretion and patients with "high" (>10 mV) stimulated chloride secretion

\begin{tabular}{|c|c|c|}
\hline & $\begin{array}{l}\text { Amiloride to } \\
\text { isoprenaline } \\
\text { difference: } \\
0-10 \mathrm{mV}\end{array}$ & $\begin{array}{l}\text { Amiloride to } \\
\text { isoprenaline } \\
\text { difference: } \\
11-25 \mathrm{mV}\end{array}$ \\
\hline Patients $\mathrm{n}$ & 15 & 7 \\
\hline $\begin{array}{l}\text { Mean amiloride to } \\
\text { isoprenaline value } \mathrm{mV}^{\dagger}\end{array}$ & $4.7(3.1)$ & $19.1(4.9)$ \\
\hline Mean age yrs & 25.5 & 32.1 \\
\hline Mean FEV1 \% pred & $48.3^{*}(17)$ & $67.7 *(19.2)$ \\
\hline Mean FVC \% pred & $68.1(15.3)$ & $75.7(9.9)$ \\
\hline $\begin{array}{l}\text { Patients with } P \text {. aeruginosa } \\
\text { colonization } \%\end{array}$ & 75 & 86 \\
\hline $\begin{array}{l}\text { i.v. antibiotics for } \\
\text { infective exacerbation } \\
\text { in last } 12 \text { months } \mathrm{n}\end{array}$ & $\begin{array}{c}52 \\
\text { (mean } 3.5 \text { per } \\
\text { patient) }\end{array}$ & $\begin{array}{c}14 \\
\text { (mean } 2 \text { per } \\
\text { patient) }\end{array}$ \\
\hline $\begin{array}{l}\text { Mean Northern } \\
\text { radiological score }\end{array}$ & $7.07 \ddagger(2.02)$ & $4.14 \ddagger(1.6)$ \\
\hline
\end{tabular}

Values in parentheses are standard deviations, unless otherwise indicated. ${ }^{\dagger}$ the highest amiloride to isoprenaline value from repeated measurements in each patient is used as that patient's value. $*: \mathrm{p}<0.05, \sharp: \mathrm{p}=0.03$, with t-test. For definitions, see legend to table 3 . 


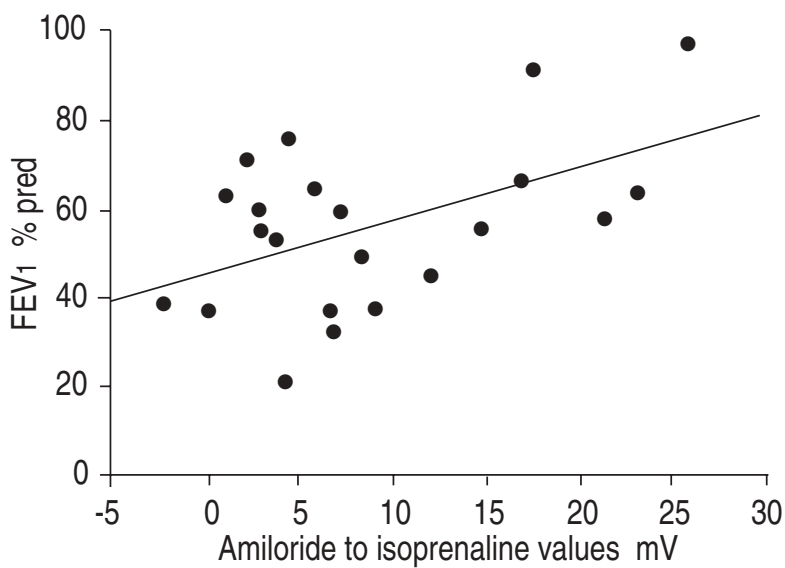

Fig. 1. - Correlation between lung function, as represented by forced expiratory volume in one second (FEV 1$) \%$ pred, and ability to secrete chloride, as measured by amiloride to isoprenaline values. $r=0.48$ on linear regression; $\mathrm{p}=0.02$ on Pearson's Moment Product correlation.
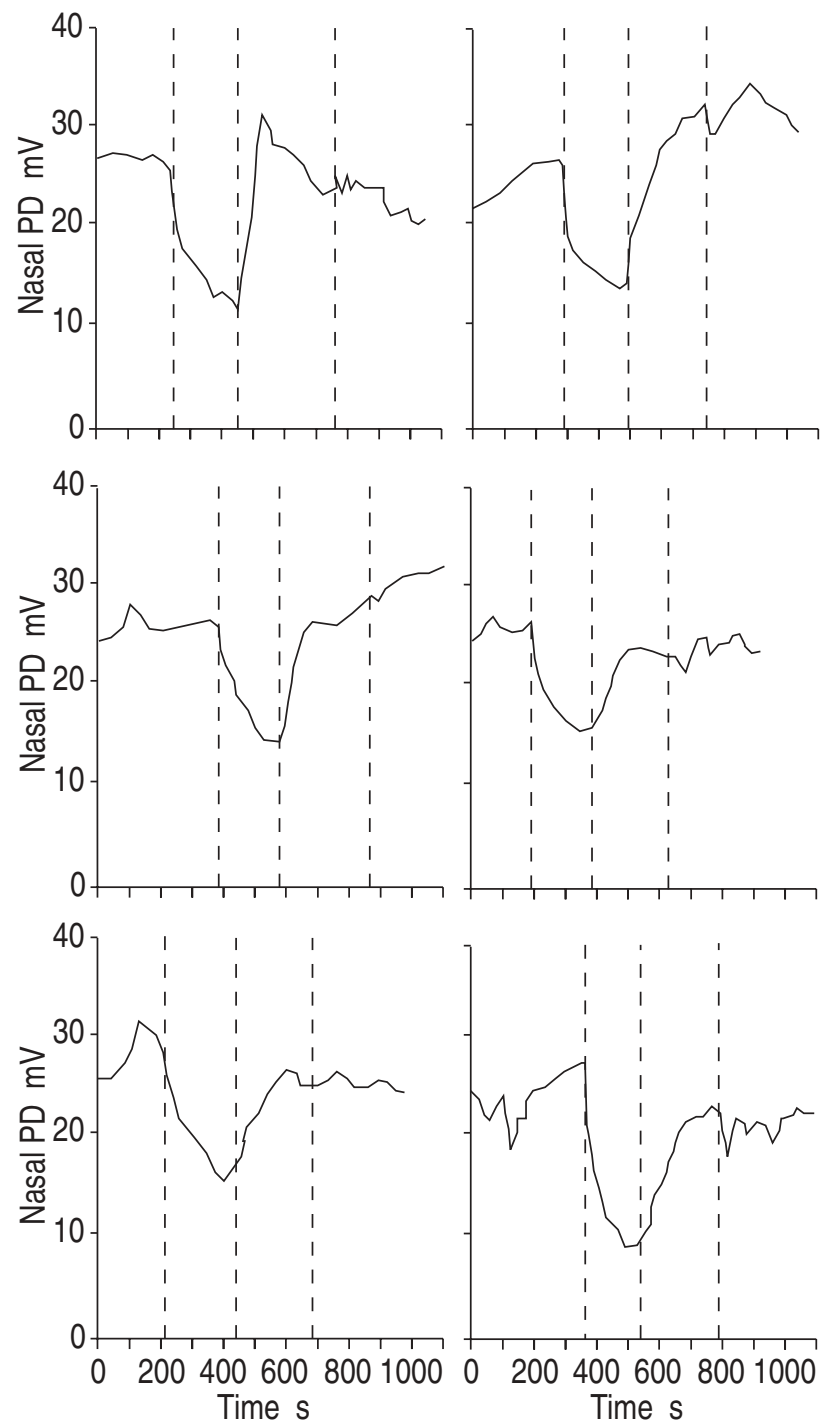

Fig. 2. - Repeated measurements of potential difference (PD) in a nostril (left) in one patient classed as a "high" secretor on different days (1-7 days apart), demonstrating reproducibility of increased chloride secretion and low baseline PD. Graphs show PD values for each second with sequential perfusion with solutions of amiloride in buffer, amiloride in low chloride and, finally amiloride and isoprenaline. The vertical lines show the start of each perfusion.
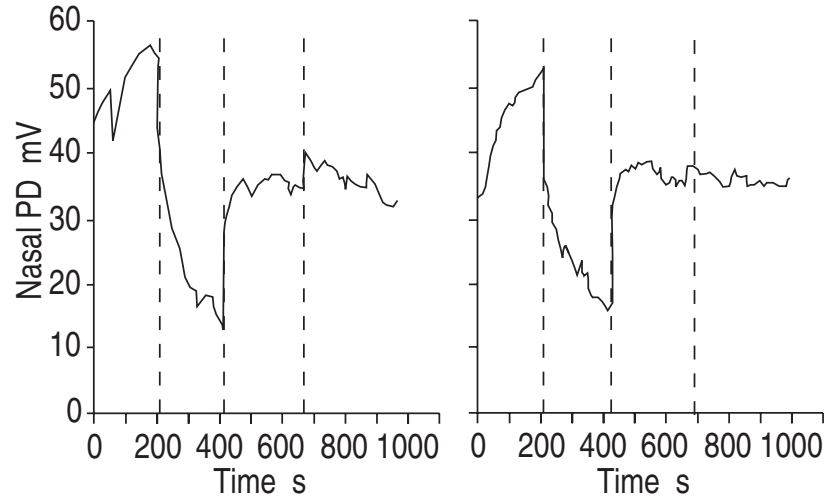

Fig. 3. - Potential difference (PD) measurements in the right nostril of one patient with $\Delta \mathrm{F} 508 / \Delta \mathrm{F} 508$, classed as a "high" secretor, 9 days apart. Graphs show PD values as described in the legend to figure 2 .

\section{Reproducibility of high chloride secretion}

In order to examine if high amiloride to isoprenaline values were reproducible, we randomly chose three patients within each of the "high" and "low" chloride secretor groups and measured their nasal PD on 12 separate occasions, each measurement separated by 1-7 days. From our repeated measurements, we found that although there was some variability over time within each individual, patients who were "high chloride secretors" tended to maintain their high secretion nasal PD profile over time (fig. 2).

The mean of all 24 nasal PD measurements (right and left nostrils) in "high" chloride secretors was statistically significantly different to that of "low" secretors (mean (SD) of 11.2 (7.6), 10.5 (6.5) and 10.5 (4.8) compared to $-1.4(5.1), 1.1(5.4)$ and $0.4(2.9) \mathrm{mV} ; \mathrm{p}<0.0001, \mathrm{t}-$ test; figs. 2 and 3 ).

\section{Discussion}

Nasal PD measurement has been put forward over recent years as a method of measuring CFTR function in $\mathrm{CF}$ patients. There is some evidence that it may be useful in diagnosing $\mathrm{CF}$ patients [10] and, recently, as an efficacy measure of gene expression in clinical studies of gene therapy for CF lung disease [14-16]. Several points arose from our studies in relation to the use of nasal PD measurements.

Firstly, there is a statistically significant correlation between the genotype of a patient and the PD response to chloride secretion stimulation. We divided the genotypes into two groups as there were not enough patients of each genotype to test individual genotype relationships with PD measurements. These groups reflected the postulated subcellular localization and residual functional capacity of the CFTR protein. The results showed that some $\mathrm{CF}$ patients have the ability to secrete chloride when stimulated with chloride secretagogue and the amount of chloride secretion (measured as amiloride to isoprenaline values) correlated with the type of CFTR mutation of the patients. In addition, even for the commonest genotype $(\Delta F 508 / \Delta F 508)$, we found some consistent "high secretors" (fig. 3). These findings have implications for the use of PD measurements 
to measure functional correction in novel corrective therapies. A small functional correction could be masked by a large basal chloride secretion, but on the other hand, an apparent correction could be due to an underlying ability to secrete chloride. It is thus imperative that PDs should be measured repeatedly over a period of time to detect the highest amiloride to isoprenaline value.

Secondly, in our study, we found a group of CF patients who appeared to be "high" secretors (amiloride to isoprenaline values $>10 \mathrm{mV}$ ). Although we found no correlation between CFTR mutation types (genotype Groups 1 and 2, see above) and clinical status of the patients, when the patients were rearranged into groups of "high" and "low" chloride secretors, the "high" secretors had significantly better lung function as seen by better FEV1 and also a significantly better radiological score. This suggests that lung function is influenced by net chloride secretion, which in turn depends not only on $\mathrm{CF}$ genotype, but also on processing of the mutant CFTR protein and on alternative parallel chloride secreting mechanisms. Indeed, three patients with $\Delta F 508 / \Delta F 508$ genotype, in whom little residual chloride secretion is expected, showed "high" chloride secretion (figure 3 shows the PD profile for one of these patients). All three patients were relatively well clinically, one aged $31 \mathrm{yrs}$ with FEV1 91\% pred, BMI 22, another aged 31 yrs with FEV1 39\% pred, BMI 20, and the last, aged 29 yrs, had an FEV1 of 55\% pred and BMI 23. All, however, were pancreatic insufficient and were colonized by Pseudomonas spp.

The only two pancreatic sufficient subjects were those who also showed the two highest values for chloride

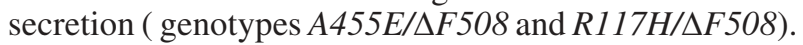
These patients had FEV1 of 95 and $60 \%$ pred, respectively. This supports suggestions from other groups that maintenance of pancreatic exocrine function requires a lower level of chloride secretion than maintenance of normal lung function.

The main limitations of the present study are the small number of patients and the different numbers of patients in the two genotype groups. We were constrained mainly by numbers of patients having different genotypes within a limited geographical area. There is a case for studying larger numbers of patients particularly within the $\Delta F 508 / \Delta F 508$ group to determine if chloride secretion could be used as a prognostic factor in the clinical course of the disease.

In conclusion, we found that there is a correlation between chloride secretion as measured by stimulated transepithelial nasal potential difference and cystic fibrosis genotype. Patients with mutations that retained an apical cystic fibrosis transmembrane regulator protein were more likely to show partial chloride secretion. Across these genotype groups, there was a smaller group of patients that appeared to have a larger $(>10$ $\mathrm{mV}$ amiloride to isoprenaline values on nasal potential difference) capacity to secrete chloride and this correlated with a better percentage predicted forced expiratory volume in one second and chest radiograph score.

Acknowledgements: The authors would like to thank B. Stack, S. Hempsey and J. Young for help with data collection and recruitment of patients from Glasgow.

\section{References}

1. Stutts M, Canessa C, Olsen J, et al. CFTR as a cAMP dependent regulator of sodium channels. Science 1995; 269: 847-850.

2. Johansen HK, Nir M, Hoiby N, Koch C, Schwartz M. Severity of $\mathrm{CF}$ in patients homozygous and heterozygous for $\Delta$ F508 mutation. Lancet 1991; 27: 665-669.

3. Kerem E, Corey M, Kerem BS, et al. The relationship between genotype and phenotype in CF- analysis of the most common mutation in $\Delta \mathrm{F} 508 . N \mathrm{Engl} \mathrm{J}$ Med 1990. 323; 22: 1517-1522.

4. Gan KH, Veeze $\mathrm{H}$, van den Ouweland A, et al. A CF mutation associated with mild lung disease. $N$ Engl $J$ Med 1995; 333: 95-99.

5. Borgo, Gasparini P, Bonizzato A, Mastella G, Pignatti PF. CF: the $\triangle F 508$ mutation does not lead to an exceptionally severe phenotype. A cohort study. Eur J Pediatr 1993; 152: 1006-1011.

6. The CF Genotype-phenotype consortium. Correlation between genotype and phenotype in patient with CF. $N$ Engl J Med 1993; 329: 1308-1313.

7. Veeze H, Halley D, Bijman J, Jongste J, Jonge H, Sinaasappel M. Determinants of mild clinical symptoms in CF patients. J Clin Invest 1994 93: 461-466

8. Knowles M, Paradiso A, Bouchier R. In vivo nasal PD: techniques and protocols for assessing efficacy of gene transfer in CF. Human Gene Ther 1995; 6: 445-455.

9. Middleton $\mathrm{P}$, Geddes D, Alton E. Protocols for in vivo measurement of the ion transport defects in $\mathrm{CF}$ nasal epithelium. Eur Respir J 1994; 7: 2050-2056.

10. Alton E, Currie D, Logan Sinclair R, Warner J, Hodsaon M, Geddes D. Nasal PD: a clinical diagnostic test for CF. Eur Respir J 1990; 3: 922-926.

11. McLachlan G, Ho LP, Davidson Smith H, et al. Laboratory and clinical studies in support of CF gene therapy using pCMV-CFTR/DOTAP. Gene Therapy 1996; 3: 1113-1123.

12. Welsh M, Smith A. Molecular mechanism of CFTR channel dysfunction in CF. Cell 1993; 73: 1251-1254.

13. Conway SP, Pond MN, Bowler I, et al. The chest radiograph in cystic fibrosis: a new scoring system compared with the Chrispin Norman and Brasfield Scores. Thorax 1994; 49: 860-862.

14. Caplen N, Alton E, Middleton P, et al. Liposome mediated CFTR gene transfer to the nasal epithelium of patients with CF. Nat Med 1995; 1: 39-47.

15. Zabner J, Couture L, Gregory R, Graham S, Smith A, Welsh M. Adenovirus mediated gene transfer transiently corrects the chloride transport defect in nasal epithelia of patients with CF. Cell 1993; 75: 207-216.

16. Knowles M, Hohneker K, Zhou Z, et al. A controlled study of adenoviral mediated gene transfer in the nasal epithelium of patients with CF. $N$ Engl J Med 1995; 333: 823-831. 